\title{
Expression of Bcl-2 and microRNAs in cardiac tissues of patients with dilated cardiomyopathy
}

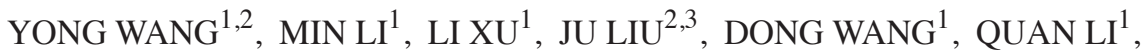 \\ LILI WANG $^{1}$, PEIJIE LI ${ }^{1}$, SHANLIANG CHEN ${ }^{1}$ and TIANQI LIU ${ }^{1}$ \\ ${ }^{1}$ Department of Cardiovascular Surgery, Shandong Provincial Qianfoshan Hospital, Shandong University, \\ Jinan, Shandong 250014; ${ }^{2}$ Weifang Medical College, Weifang, Shandong $261031 ;{ }^{3}$ Medical Research Center, \\ Shandong Provincial Qianfoshan Hospital, Shandong University, Jinan, Shandong 250014, P.R. China
}

Received October 26, 2015; Accepted November 1, 2016

DOI: $10.3892 / \mathrm{mmr} .2016 .5977$

\begin{abstract}
Dilated cardiomyopathy (DCM) is associated with sudden cardiac death and heart failure, resulting in a significant medical burden. The mechanisms underlying the pathogenesis of DCM remain elusive. In the present study, human cardiac tissues from patients with DCM and healthy donors were collected and their pathology was examined. The expression levels of apoptosis regulator $\mathrm{Bcl}-2$ and fibrosis-associated microRNAs were also evaluated. Extensive myocardial fibrosis and apoptosis in DCM cardiac tissues was observed. As demonstrated by western blotting, reverse transcription-quantitative polymerase chain reaction and immunohistochemistry, the expression of $\mathrm{Bcl}-2$ was significantly increased in the apex, and the left and right ventricle of the heart in patients with DCM. In the specified locations, it was identified that miR-21 was upregulated, while members of miR-29 family (miR-29a, miR-29b and miR-29c) and miR-133 family (miR-133a and miR-133b) were downregulated. The present study suggested that Bcl-2 and specific microRNAs may be involved in DCM pathogenesis, with a potential implication as therapeutic targets.
\end{abstract}

\section{Introduction}

Dilated cardiomyopathy (DCM) is characterized by left ventricular dilation and systolic dysfunction with an ejection fraction of $<50 \%$ (1). DCM is associated with sudden

Correspondence to: Dr Tianqi Liu, Department of Cardiovascular Surgery, Shandong Provincial Qianfoshan Hospital, Shandong University, 16766 Jingshi Road, Jinan, Shandong 250014, P.R. China E-mail: tianqiliu12@aliyun.com

Dr Ju Liu, Medical Research Center, Shandong Provincial Qianfoshan Hospital, Shandong University, 16766 Jingshi Road, Jinan, Shandong 250014, P.R. China

E-mail: ju.liu@sdu.edu.cn

Key words: dilated cardiomyopathy, fibrosis, apoptosis, Bcl-2, microRNA cardiac death and heart failure, resulting in a significant medical burden due to the high rate of hospital admissions and the potential requirement for heart transplantation (2). Despite therapeutic advances, the prevalence of DCM was 1 in 2,500-3,000 and the 5-year mortality remained $20 \%$ in 2010 (2,3). Patients with DCM may also develop diastolic dysfunction and impaired right ventricular function. Genetic inheritance, myocarditis, alcohol intake and toxic effects from medications may contribute to the development and progression of DCM (2).

Cardiomyocyte apoptosis and fibrosis are the major pathological changes that occur in DCM. Apoptosis is an actively regulated process that includes DNA fragmentation, membrane blebbing, cell shrinking and chromatin condensation (4). Fibrosis is the formation of excess fibrous tissues or scar tissue, which is characterized by the net accumulation of extracellular matrix proteins in the cardiac interstitium (5). In the hearts of patients with DCM, apoptosis of cardiomyocytes leads to the increase of the intercellular space, where it is filled by granulation tissue, composed of macrophages, endothelial cells and fibroblasts $(6,7)$. In addition, cardiac fibroblasts excessively produce extracellular matrix (ECM), leading to fibrosis and increasing tissue stiffness.

B-cell lymphoma-2 (Bcl-2) is an important antiapoptotic protein $(8,9)$. It was isolated from the $t(14 ; 18)$ chromosomal breakpoint in follicular B-cell lymphoma (10). The Bcl-2 protein has been demonstrated to bind to Bak, Bax, Bcl-xL, Bcl-xS, mcl-1 and a variety of other intracellular proteins (11). The relative levels and competing dimerizations between Bcl-2 and its binding proteins determine the potential of a cell to undergo apoptosis (12). Overexpression of Bcl-2 in transgenic models leads to accumulation of cells, due to evasion of cell death mechanisms (13). Induction of apoptosis by diverse stimuli, including radiation, hyperthermia, growth factor withdrawal and multiple classes of chemotherapeutic agents, is inhibited by Bcl-2 in vitro (14). The inhibitory effects are proportional to the level of Bcl-2 expression (14). In addition, an increase in the expression levels of the Bcl-2 family proteins was observed in the end stage of heart failure (15). However, the role of Bcl-2 in DCM remains to be thoroughly studied.

Micro (mi)RNAs are 22 nucleotides in length and serve important regulatory roles by targeting messenger RNAs for 
cleavage or translational repression via RNA silencing (16). They are the most abundant class of regulatory molecules for the expression of protein-coding genes in response to pathological stimuli (17). Genome-wide profiling of miRNAs demonstrated that 43 miRNAs were differentially expressed during human heart failure (18). The level of miRNAs in blood circulation may function as markers for DCM (19). Specific miRNAs, including miR-21, may regulate $\mathrm{Bcl}-2$ and mediate fibrosis (20). Therefore, the expression of miRNAs in cardiac tissues may be associated with the pathogenesis of DCM.

In the present study, human hearts from three DCM patients and three healthy donors were collected. Myocardial fibrosis and apoptosis of the cardiac tissues was evaluated, and the mRNA and protein expression levels of Bcl-2 were examined. In addition, the expression of three miRNAs and their family members (miR-21; miR-29a, miR-29b and miR-29c; miR-133a and miR-133b) were examined in normal and DCM cardiac tissues. The results of the present study suggested that Bcl-2 and specific miRNAs are involved in human DCM.

\section{Materials and methods}

Human cardiac tissues. Experiments involving human tissues complied with the Declaration of Helsinki and were approved by the Shandong Provincial Qianfoshan Hospital Ethical Review Board (Jinan, China; no. S074). Human hearts were collected from three patients with DCM-caused

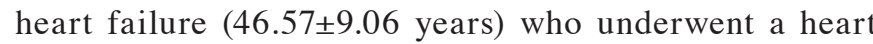
transplantation. The normal cardiac tissues were from three adults (32.0 \pm 7.16 years old) with accidental traumatic brain death, who had no medical evidence of cardiac disease. All patients were functionally classified according to the New York Heart Association criteria. The diagnosis of DCM was based on the World Health Organization/International Society and Federation Cardiology definition (21). Clinical history, electrocardiogram, echocardiography, hemodynamic studies and coronary angiography data were measured for all patients (Table I). Transmural samples were taken from near the apex, septum, and left and right ventricle, and were stored in frozen liquid nitrogen or fixed in $10 \%$ buffered formalin for analysis.

Masson's trichrome staining. Masson's trichrome staining was performed as previously described (22). The frozen cardiac tissues were cut into $6 \mu \mathrm{m}$ transverse sections, stained with Weigert's iron hematoxylin for $10 \mathrm{~min}$ at room temperature, then washed and stained in $1 \%$ ponceau-acetic acid solution (equal volumes of $0.5 \%$ ponceau $2 \mathrm{R}$ in $1 \%$ acetic acid and $0.5 \%$ acid fuchsin in $1 \%$ acetic acid) for $5 \mathrm{~min}$ at room temperature. Following washing, the sections were incubated with $1 \%$ phosphomolybdic acid for 5 min and counterstained with light green. The images were captured under a FSX100 microscope (Olympus Corporation, Tokyo, Japan) and analyzed using Image-Pro Plus Software (version 6.0; Media Cybernetics, Inc., Rockville, MD, USA).

Terminal deoxynucleotidyl-mediated dUTP nick end labeling (TUNEL) assay. TUNEL (EMD Millipore, Billerica, MA, USA) was used to detect the apoptosis of cardiac tissues. The frozen tissue sections were pretreated with $20 \mu \mathrm{g} / \mathrm{ml}$ proteinase
K (EMD Millipore) for $15 \mathrm{~min}$ at room temperature, and the endogenous peroxidase activity was eliminated using $3 \%$ hydrogen peroxide in phosphate-buffered saline (PBS; $5 \mathrm{~min}$ at room temperature). Following washing with equilibration buffer from the kit, the tissue sections were incubated with the reaction mixture from the kit for $50 \mathrm{~min}$ at $37^{\circ} \mathrm{C}$. Following the anti-digoxigenin-peroxidase reaction for $30 \mathrm{~min}$, the positive cells were visualized by diaminobenzidine (Dako, Glostrup, Denmark) and the slides were counterstained with hematoxylin. The number of positively stained cells and total cells were calculated under a FSX100 microscope (Olympus Corporation).

Reverse transcription-quantitative polymerase chain reaction $(R T-q P C R)$. RNA isolation was performed using the RNeasy Mini kit (Qiagen GmbH, Hilden, Germany). The cDNA was synthesized with the High Capacity RNA-to-cDNA kit (Applied Biosystems; Thermo Fisher Scientific, Inc., Waltham, MA, USA). qPCR was performed using a ViiA7 Real-Time PCR system (Applied Biosystems; Thermo Fisher Scientific, Inc.), according to the manufacturer's instructions. Reactions were performed at $50^{\circ} \mathrm{C}$ for $2 \mathrm{~min}$ and $95^{\circ} \mathrm{C}$ for $2 \mathrm{~min}$, followed by 40 cycles of $95^{\circ} \mathrm{C}$ for $15 \mathrm{sec}$ and $60^{\circ} \mathrm{C}$ for $1 \mathrm{~min}$. The relative Bcl-2 expression was calculated using GAPDH as an endogenous internal control. The primer sequences were as follows: Bcl-2, forward: 5'-ATGGGCTGGGCATAGAGAC-3' and reverse: 5'-TTGAAGCCATGTGTCCACTC-3'; GAPDH, forward: 5'-AGCTGAACGGGAAGCTCACT-3' and reverse: 5'-TAGGTCCACCACTGACACGTTG-3'.

Additionally, RT-qPCR was performed with primers specific for human miR-21 (MIMAT0004494), miR-29a (MIMAT0000086), miR-29b (MIMAT0000100), miR-29c (MI MAT0000681), miR-133a (MIMAT0000427) and miR-133b (MIMAT0000770). All primers were designed by Tiangen Biotech Co., Ltd. (Beijing, China). Each reaction was repeated in triplicate, and the experiments were repeated to confirm reproducibility.

Immunohistochemical analysis. Tissue sections were deparaffinized in xylene and rehydrated through graded alcohol washes, prior to incubating with $1 \% \mathrm{H}_{2} \mathrm{O}_{2}$ in methanol for $10 \mathrm{~min}$ at room temperature to inhibit endogenous peroxidase activity. Following washing with PBS, the tissue sections were incubated with a mouse anti-Bcl-2 antibody (1:100; catalog no. ab692; Abcam, Cambridge, MA, USA) in a humid chamber at $4^{\circ} \mathrm{C}$ overnight, followed by incubation with a biotinylated anti-mouse secondary antibody (1:200; catalog no. ZDR-5307; Beijing Zhongshan Jinqiao Biotechnology Co., Ltd., Beijing, China) for $30 \mathrm{~min}$ at room temperature. Diaminobenzidine tetrahydrochloride was used to visualize the antibody binding, and hematoxylin counterstain was performed prior to the addition of coverslips. Omission of the primary antibody resulted in a lack of specific staining, therefore, serving as the negative control. Specimens were examined with an FSX100 microscope (Olympus Corporation). A total of 20 images of each section were captured at a magnification of $\mathrm{x} 20$. The expression of Bcl-2 was analyzed using Image-Pro Plus software (version 6.0; Media Cybernetics, Inc.). The positive expression of $\mathrm{Bcl}-2$ was determined by the ratio of the positive area to the total area. 
Western blot analysis. Tissue samples were lysed in ice-cold RIPA buffer [20 mM Tris (pH 7.5), $150 \mathrm{mM} \mathrm{NaCl}, 50 \mathrm{mM}$ NaF, $1 \%$ NP-40, $0.1 \%$ deoxycholate, $0.1 \%$ SDS and $1 \mathrm{mM}$ EDTA, supplemented with $1 \mathrm{mM}$ PMSF and $1 \mu \mathrm{g} / \mathrm{ml}$ leupeptin]. The protein concentration was determined using Bio-Rad DC Protein assay (Bio-Rad Laboratories, Inc., Hercules, CA, USA). An equal quantity ( $20 \mathrm{mg}$ ) of the samples were fractionated by SDS-PAGE and transferred to a polyvinylidene difluoride membrane (GE Healthcare Life Sciences, Chalfont, UK). The membranes were blocked for $1 \mathrm{~h}$ at room temperature with 5\% non-fat dry milk in Tris-buffered saline containing $0.05 \%$ Tween, and were subsequently incubated overnight at $4^{\circ} \mathrm{C}$ with a polyclonal rabbit anti-Bcl-2 antibody (1:1,000; catalog no. ab59348; Abcam) or a rabbit GAPDH polyclonal antibody (1:3,000, catalog no. 10494-1-AP; ProteinTech Group, Inc., Chicago, IL, USA). Following washing three times, the membranes were incubated with a horseradish peroxidase-labeled goat anti-rabbit IgG (1:5,000; catalog no. BA1054; Boster Systems, Inc., Pleasanton, CA, USA), and the immunoblots were visualized using enhanced chemiluminescence Western Blotting Luminol reagent (Santa Cruz Biotechnology, Inc., Dallas, TX, USA). The intensity of the bands from each protein under investigation was quantified using Image J software version 1.48 (National Institutes of Health, Bethesda, MD, USA).

Statistical analysis. The data are presented as the mean \pm standard error of the mean. Statistical significance was assessed using paired Student's $t$-tests. $\mathrm{P}<0.05$ was considered to indicate a statistically significant difference. All statistical analyses were performed using SPSS software (version, 18.0; SPSS, Inc., Chicago, IL, USA).

\section{Results}

Fibrosis and apoptosis is increased in cardiac tissues of patients with DCM. Pathological changes in myocardial tissue of DCM patients were examined using Masson's trichrome staining and TUNEL. Masson's trichrome staining demonstrated that collagen was appropriately arranged among cardiomyocytes in the normal group, while it was increased and disorganized in the DCM group (data not shown). The size of the nucleus was uniform in the normal cardiomyocytes, but irregular in the cardiomyocytes from patients with DCM (data not shown). In the DCM group, the collagen volume fractions, a morphometric measure of the percentage of interstitial collagen, were significantly higher when compared with the normal group in the apex, and the left and right ventricles (Fig. $1 \mathrm{~A} ; 4.23 \pm 1.28$ vs. $9.84 \pm 1.39 \%, \mathrm{P}<0.05 ; 3.89 \pm 0.99$ vs. $10.84 \pm 1.65 \%, \mathrm{P}<0.05 ; 5.19 \pm 1.36$ vs. $11.09 \pm 1.07 \%, \mathrm{P}<0.05$, respectively); however, were not significantly increased in the septum (Fig. $1 \mathrm{~A} ; 3.48 \pm 1.11$ vs. $4.21 \pm 1.31, \mathrm{P}=0.69$ ). TUNEL was used to label apoptotic cells. The results presented in the present study indicated a greater TUNEL staining in the tissue sections of apex, and left and right ventricles of the DCM group when compared with the normal group (Fig. 1B). The myocardial apoptosis index, denoted as the percentage of TUNEL positive cells, was significantly higher in the apex, and the left and right ventricle of the DCM group when compared with the normal group (Fig. 1B; $8.25 \pm 1.93$ vs. $43.6 \pm 7.31 \%$,
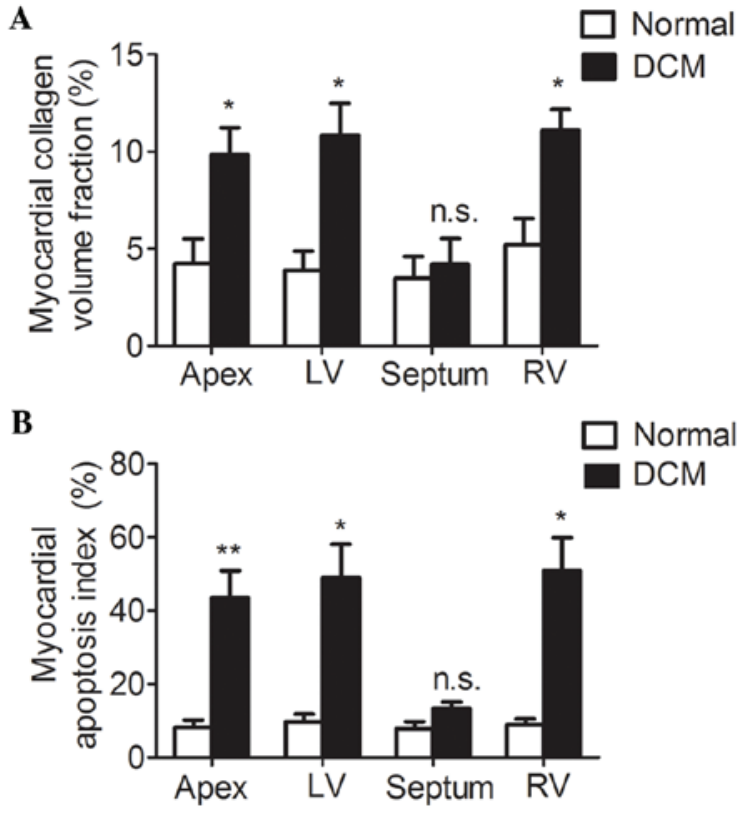

Figure 1. Myocardial fibrosis and apoptosis in patients with DCM (A) Analyses of myocardial collagen volume fraction in the heart apex, LV, septum and RV. (B) Analyses of Tunel positive cells/apoptotic index. The data are presented as the mean \pm standard error of the mean $(n=3 ; n . s$., non-significant; ${ }^{\mathrm{P}}<0.05 ;{ }^{* *} \mathrm{P}<0.01$ compared with the Normal group). $\mathrm{LV}$, left ventricle; RV, right ventricle; DCM, dilated cardiomyopathy.

$\mathrm{P}<0.01 ; 9.70 \pm 2.13$ vs. $49.02 \pm 9.08 \%, \mathrm{P}<0.05 ; 8.92 \pm 1.56$ vs. $50.89 \pm 8.99 \%, \mathrm{P}<0.05$, respectively). The myocardial apoptosis index in the septum presented no statistical difference between the two groups (Fig. $1 \mathrm{~B} ; 7.92 \pm 1.83$ vs. $13.35 \pm 1.76 \%, \mathrm{P}=0.09$ ). Therefore, cardiac fibrosis and apoptosis were significantly increased in apex, and the left and right ventricle of patients with DCM when compared with the normal control group.

Expression of $\mathrm{Bcl}-2$ is upregulated in cardiac tissues from patients with DCM. Bcl-2 is an inner mitochondrial membrane protein that blocks programmed cell death. The present study analyzed the expression levels of Bcl-2 in cardiac tissue from human samples. Western blot analysis demonstrated that Bcl-2 was significantly increased in the apex, and the left and right ventricle of DCM samples when compared with the normal group (Fig. 2A), which was confirmed by densitometry analysis (Fig. 2B; $0.6623 \pm 0.0824$ vs. $1.1991 \pm 0.1623$, $\mathrm{P}<0.05$; $1.0925 \pm 0.1262$ vs. $1.8084 \pm 0.1831, \mathrm{P}<0.05 ; 0.6459 \pm 0.1029$ vs. $1.0994 \pm 0.1243, \mathrm{P}<0.05$, respectively). The mRNA expression of $\mathrm{Bcl}-2$ was determined by RT-qPCR. This indicated that Bcl-2 was significantly upregulated in the apex, and the left and right ventricle of the patients with DCM compared with the normal group (Fig. $2 \mathrm{C} ; 0.0007 \pm 0.0002$ vs. $0.0018 \pm 0.0003, \mathrm{P}<0.05$; $0.0007 \pm 0.0003$ vs. $0.0021 \pm 0.0002, \mathrm{P}<0.01 ; 0.0006 \pm 0.0001$ vs. $0.0019 \pm 0.0004, \mathrm{P}<0.05$, respectively). No significant difference was observed in either the mRNA or protein expression level of Bcl-2 in the septum (Fig. 2B, $\mathrm{P}=0.95$; Fig. 2C, $\mathrm{P}=0.60$, respectively). Immunohistochemistry of Bcl-2 was performed on the cardiac tissues (Fig. 3A) and the staining was quantified by the intensity of cytoplasmic staining and percentage of expression. Each analysis indicated that the expression of $\mathrm{Bcl}-2$ was increased in the apex, and the left and right ventricle 
Table I. Clinical characteristics of the patient groups.

\begin{tabular}{lcc}
\hline Characteristic & $\begin{array}{c}\text { Normal group } \\
(\mathrm{n}=3)\end{array}$ & $\begin{array}{c}\text { DCM group } \\
(\mathrm{n}=3)\end{array}$ \\
\hline LVEF $(\%)$ & $68.12 \pm 5.09$ & $29.06 \pm 5.47^{\mathrm{a}}$ \\
LVEDD $(\mathrm{cm})$ & $2.79 \pm 0.84$ & $6.98 \pm 0.68^{\mathrm{a}}$ \\
CO $(1 / \mathrm{min})$ & $5.38 \pm 1.13$ & $2.07 \pm 0.83^{\mathrm{a}}$ \\
PCWP $(\mathrm{mmHg})$ & $9.59 \pm 1.98$ & $26.13 \pm 9.06^{\mathrm{a}}$ \\
NT-ProBNP $(\mathrm{ng} / \mathrm{ml})$ & $109 \pm 19.39$ & $1709.19 \pm 78.97^{\mathrm{a}}$ \\
hs-CRP $(\mathrm{mg} / \mathrm{l})$ & $0.61 \pm 0.14$ & $1709.19 \pm 78.97^{\mathrm{a}}$ \\
\hline
\end{tabular}

${ }^{\text {aP }}<0.001$ compared with the normal group. LVEF, left ventricular ejection fraction; LVEDD, left ventricular end-diastolic diameter; $\mathrm{CO}$, cardiac output; PCWP, pulmonary capillary wedge pressure; NT-ProBNP, n-terminal pro-brain natriuretic peptide; hs-CRP, high sensitivity c-reaction protein; DCM, dilated cardiomyopathy.

$(\mathrm{P}<0.01)$, however, not in the septum $(\mathrm{P}=0.08, \mathrm{P}=0.07$, respectively) of the DCM cardiac tissues (Fig. 3B and C). These results indicated that $\mathrm{Bcl}-2$ was upregulated in the majority of regions of the ventricles, with the exception except the septum.

Expression of miRNAs is differentially regulated in cardiac tissues from patients with DCM. The expression levels of miR-21, and members of the miR-29 and miR-133 families were examined in the apex, septum, and left and right ventricles using RT-qPCR. miR-21 was significantly upregulated in the apex, and the left and right ventricles of patients with DCM when compared with the normal group (Fig. 4A; $\mathrm{P}<0.05$, $\mathrm{P}<0.01, \mathrm{P}<0.05$, respectively). In these locations, the expression levels of miR-29a, miR-29b, miR-29c, miR-133a and miR-133b were significantly downregulated in comparison with the normal group (Fig. 4B-F; miR-29a: $\mathrm{P}<0.05, \mathrm{P}<0.01, \mathrm{P}<0.01$, respectively; miR-29b: $\mathrm{P}<0.05, \mathrm{P}<0.05, \mathrm{P}<0.05$, respectively; miR-29c: $\mathrm{P}<0.05, \mathrm{P}<0.05, \mathrm{P}<0.05$, respectively; miR-133a: $\mathrm{P}<0.05, \mathrm{P}<0.01, \mathrm{P}<0.05$, respectively; miR-133b: $\mathrm{P}<0.01$, $\mathrm{P}<0.01, \mathrm{P}<0.05$, respectively). In the septum, no significant difference was identified for the expression of all miRNAs between normal and DCM groups (Fig. 4A-F; miR-21, $\mathrm{P}=0.86$; miR-29a, $\mathrm{P}=0.09$; miR-29b, $\mathrm{P}=0.59$; miR-29c, $\mathrm{P}=0.55$; miR-133a, $\mathrm{P}=0.99$; miR-133b, $\mathrm{P}=0.96$ ). Thus, the expression of miRNAs is differentially regulated in DCM.

\section{Discussion}

DCM is associated with significant morbidity and premature mortality (23). The mechanisms of DCM pathogenesis remain unclear. In the present study, fibrosis and apoptosis in cardiac tissues of healthy donors and patients with DCM were examined. It was demonstrated that the expression of Bcl-2 was upregulated in the apex, and the left and right ventricles of the heart in patients with DCM. Furthermore, these results demonstrated that miRNAs are differentially regulated in the cardiac tissues of DCM patients, which are associated with the expression of $\mathrm{Bcl}-2$. Taken together, the present study suggested that dysregulation of Bcl-2 and specific miRNAs may involve in the pathogenesis of DCM.

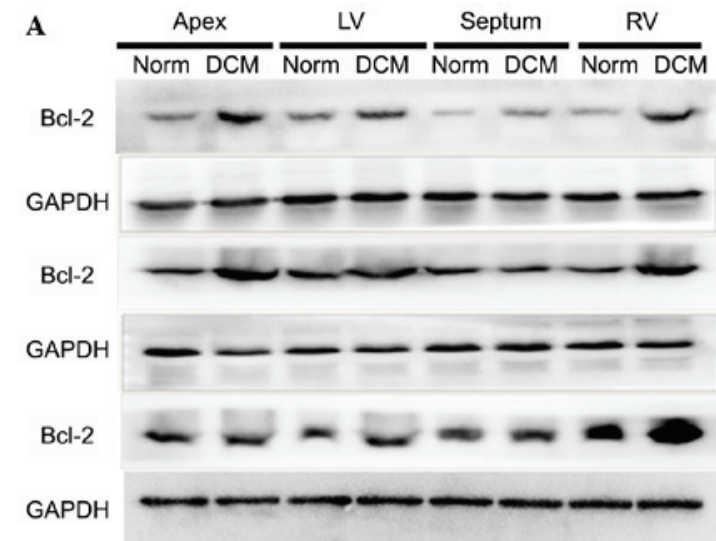

B

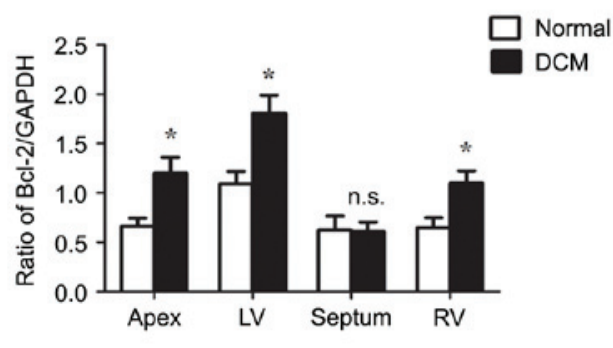

C

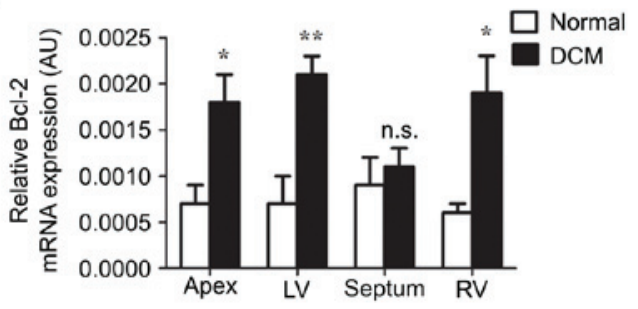

Figure 2. Protein and mRNA expression levels of Bcl-2 in human cardiac tissues. (A) Western blot analysis of Bcl-2 protein in three samples from various cardiac tissues from the normal and DCM group. (B) Densitometry analyses of the western blot analyses. (C) Reverse transcription-quantitative polymerase chain reaction analyses of the mRNA expression of Bcl-2 in the normal and DCM group. The data are presented as the mean \pm standard error of the mean ( $\mathrm{n}=3$; n.s., non-significant; ${ }^{*} \mathrm{P}<0.05$; ${ }^{* *} \mathrm{P}<0.01$ compared with the Normal group). LV, left ventricle; RV, right ventricle; Bcl-2, B-cell lymphoma 2; DCM, dilated cardiomyopathy; Norm, normal group; AU, arbitrary units.

Cardiovascular fibrosis alters myocardial stiffness and leads to ventricular dysfunction (5). Biopsy studies have demonstrated that DCM is associated with diffuse myocardial fibrosis in the ventricles (24). Unverferth et al (25) observed extensive left ventricle tissue fibrosis in patients with DCM. In the present study, it was identified that fibrosis, which is calculated by collagen content, significantly increased in the apex, and the left and right ventricles of patients with DCM when compared with the normal group. In these locations of the heart, an elevated number of TUNEL-positive cells were observed in the tissues from patients with DCM. Based on the observation of dead myocytes and replacement fibrosis, myocardial cell loss was likely attributed to tissue necrosis. However, apoptosis also occurs in human hearts under various pathological conditions, including acute myocardial infarction, arrhythmogenic right ventricular dysplasia and restenosis $(4,26,27)$. These results support that apoptosis serves a critical role in DCM. No significant change was demonstrated in fibrosis and apoptosis in the septum, suggesting that septum may be affected in a later stage compared with the ventricles and apex. 

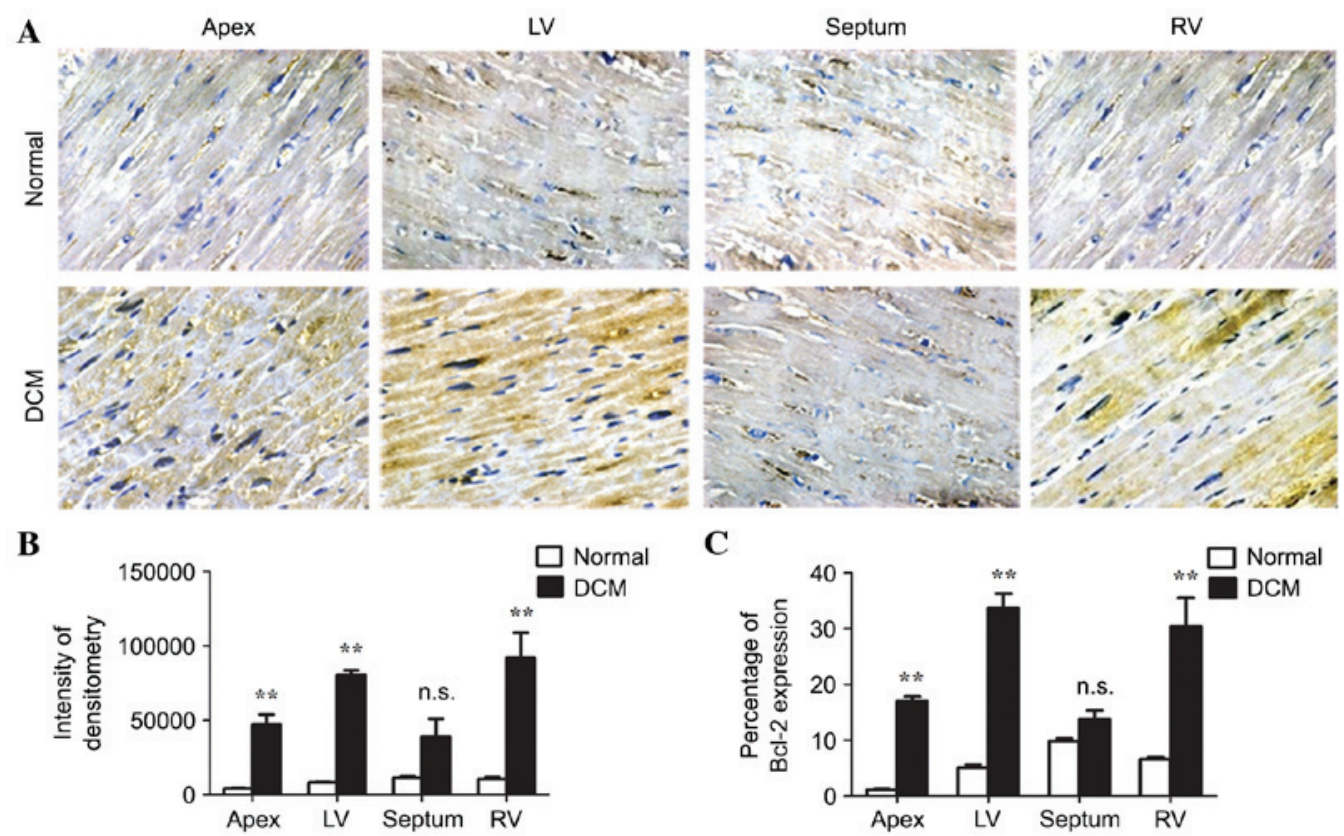

Figure 3. Immunohistochemistry of Bcl-2 on human cardiac tissues. (A) Representative images of Bcl-2 immunostaining on human cardiac tissue (magnification, $\mathrm{x} 40$ ). Analyses of (B) the intensity of densitometry and (C) the percentage of Bcl-2 expression. The data are presented as the mean \pm standard error of the mean ( $\mathrm{n}=3$; n.s., non-significant; ${ }^{*} \mathrm{P}<0.05 ;{ }^{* *} \mathrm{P}<0.01$ compared with the Normal group). LV, left ventricle; RV, right ventricle; Bcl-2, B-cell lymphoma-2; DCM, dilated cardiomyopathy.
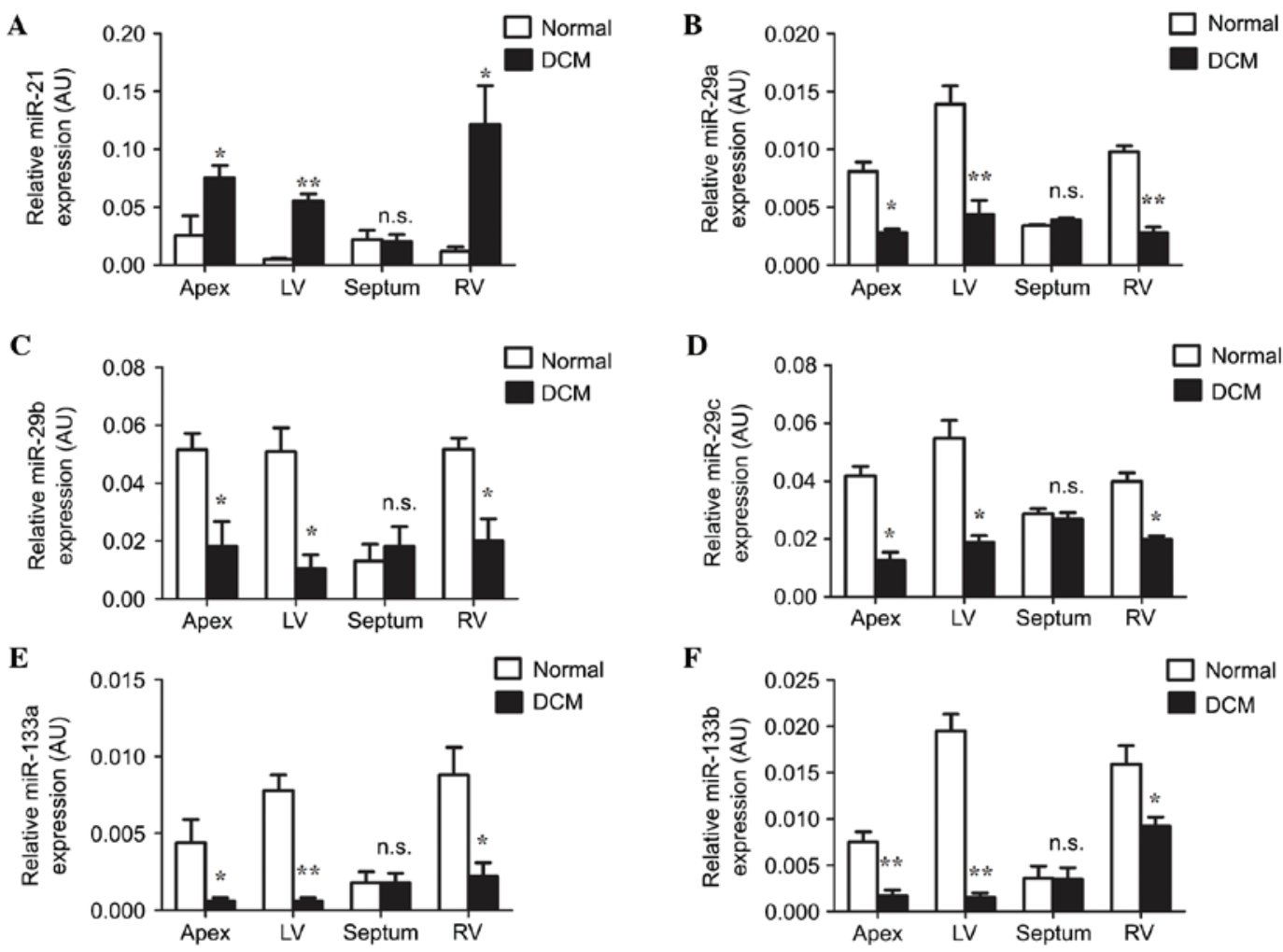

Figure 4. Reverse transcription-quantitative polymerase chain reaction analysis of microRNA expression in human cardiac tissues. The relative expression levels of (A) miR-21, (B) miR-29a, (C) miR-29b, (D) miR-29c, (E) miR-133a and (F) miR-133b were determined. The data are presented as the mean \pm standard error of the mean ( $\mathrm{n}=3$; n.s., non-significant; ${ }^{*} \mathrm{P}<0.05$; ${ }^{* *} \mathrm{P}<0.01$ compared with the Normal group). LV, left ventricle; RV, right ventricle; RT-qPCR, quantitative reverse transcriptase polymerase chain reaction; AU, arbitrary units; DCM, dilated cardiomyopathy; miR, microRNA.

$\mathrm{Bcl}-2$ is one of the primary regulators of apoptosis and the Bcl-2 proto-oncogene suppresses apoptosis (28). Using immunochemistry, western blotting and RT-qPCR, significant increases in the expression of Bcl-2 were observed in the apex, and the left and right ventricles in patients with DCM compared with the normal donors. These locations also 
demonstrated intensive fibrosis and apoptosis, suggesting that upregulation of Bcl-2 may be associated with cardiac fibrosis and apoptosis. Upregulation of this antiapoptotic protein raises the apoptotic threshold, which increases apoptosis resistance under cellular abnormalities. Although the detailed mechanisms of Bcl-2 protein function remain to be fully understood, it binds to proapoptotic proteins and inhibits channels crossing mitochondria (29). Since apoptosis is responsible for the progression of heart failure and the chronic loss of ventricular function, the increased expression of Bcl-2 may implicate the presence of a compensatory mechanism in patients with DCM.

miRNAs are endogenous, non-coding, 21-23 nucleotide RNAs that regulate gene expression by binding to the 3'untranslated region (3'UTR) of the target gene mRNAs to repress translation or induce mRNA cleavage. Increasing evidence suggests that certain miRNAs contribute to cardiac fibrosis (30). The present study identified that six miRNAs (miR-21; miR-29a, miR-29b and miR-29c; miR-133a and miR-133b) were significantly associated with the pathogenesis of DCM. miR-21 has been increasingly drawing the interests of cardiovascular research. Thum et al (31) demonstrated that upregulated expression of miR-21 enhances the signal transduction of the EPK-MAPK pathway, thereby leading to the proliferation of fibroblasts and cardiac fibrosis. In addition, silencing of the miR-21 gene inhibits the transduction of EPK-MAPK signals, thus inhibiting the fibrosis and improving cardiac functions (31). In a mouse model of ischemia-reperfusion, miR-21 regulates MMP-2 expression via a PTEN pathway during cardiac fibrosis and remodeling (32). This previous study revealed that the expression of miR-21 was higher in highly fibrotic sections of DCM tissue, which is consistent with its role in fibrosis.

miR-29 and miR-133 are also positive regulators of fibrosis (32-35). The miR-29 family of miRNAs has been demonstrated to target gene transcripts that encode ECM proteins involved in fibrotic responses, including different collagen isoforms (COL1A1, COL1A2, and COL3A1), fibrillin-1 and elastin (33). Downregulation of miR-29 induces the expression of collagens, whereas overexpression of miR-29 in fibroblasts reduces collagen expression (33). miR-29 acts as a positive regulator of fibrosis and represents a potential therapeutic target. Additionally, miR-133 is a novel molecular player in the heart. Decreased miR-133 in myocytes induces an upregulation of TGF- $\beta 1$ and TGF- $\beta$ RII proteins, which leads to enhanced collagen production and fibrosis generation (34). In addition, miR-133 positively regulates the $\beta 1 \mathrm{AR}$ pathway at multiple levels, which causes pathological cardiac hypertrophy and fibrosis $(35,36)$. In the present study, the expression levels of miR-29 and miR-133 family members were lower in DCM tissues compared with in the corresponding locations in normal heart tissue. This suggested that mechanisms of negative regulation on fibrosis through miRNAs may exist during the progression of DCM.

In conclusion, extensive myocardial fibrosis and apoptosis in human DCM cardiac tissues was observed. The expression of the apoptosis regulator Bcl-2 was increased in the apex, and the left and right ventricles in patients with DCM compared with that in normal controls. In these locations, miR-21 was upregulated, while members of miR-29 family and miR-133 family were downregulated. The present study suggested that
miRNAs are differentially regulated during cardiac fibrosis, thus they may represent a novel class of therapeutic targets for DCM.

\section{Acknowledgements}

The present study was supported by grants from The Natural Science Foundation of Shandong Province (nos. Z2006C10 and ZR2013HM028) and The National Natural Science Foundation of China (no. 81370269). The authors are grateful for the support from Shandong Taishan Scholarship awarded to Ju Liu.

\section{References}

1. Burkett EL and Hershberger RE: Clinical and genetic issues in familial dilated cardiomyopathy. J Am Coll Cardiol 45: 969-981, 2005.

2. Jefferies JL and Towbin JA: Dilated cardiomyopathy. Lancet 375: 752-762, 2010.

3. Felker GM, Thompson RE, Hare JM, Hruban RH, Clemetson DE, Howard DL, Baughman KL and Kasper EK: Underlying causes and long-term survival in patients with initially unexplained cardiomyopathy. N Engl J Med 342: 1077-1084, 2000.

4. Isner JM, Kearney M, Bortman S and Passeri J: Apoptosis in human atherosclerosis and restenosis. Circulation 91: 2703-2711, 1995.

5. Weber KT, Brilla CG and Janicki JS: Myocardial fibrosis: Functional significance and regulatory factors. Cardiovasc Res 27: 341-348, 1993.

6. Frangogiannis NG, Smith $\mathrm{CW}$ and Entman ML: The inflammatory response in myocardial infarction. Cardiovasc Res 53: 31-47, 2002.

7. Agocha A, Lee HW and Eghbali-Webb M: Hypoxia regulates basal and induced DNA synthesis and collagen type I production in human cardiac fibroblasts: Effects of transforming growth factor-beta1, thyroid hormone, angiotensin II and basic fibroblast growth factor. J Mol Cell Cardiol 29: 2233-2244, 1997.

8. Di Napoli P, Taccardi AA, Grilli A, Felaco M, Balbone A, Angelucci D, Gallina S, Calafiore AM, De Caterina R and Barsotti A: Left ventricular wall stress as a direct correlate of cardiomyocyte apoptosis in patients with severe dilated cardiomyopathy. Am Heart J 146: 1105-1111, 2003.

9. Tsipis A, Athanassiadou AM, Athanassiadou P, Kavantzas N, Agrogiannis G and Patsouris E: Apoptosis-related factors p53, bcl- 2 and the defects of force transmission in dilated cardiomyopathy. Pathol Res Pract 206: 625-630, 2010.

10. Cleary ML and Sklar J: Nucleotide sequence of a $t(14 ; 18)$ chromosomal breakpoint in follicular lymphoma and demonstration of a breakpoint-cluster region near a transcriptionally active locus on chromosome 18. Proc Natl Acad Sci USA 82: 7439-7443, 1985.

11. Sato T, Hanada M, Bodrug S, Irie S, Iwama N, Boise LH, Thompson CB, Golemis E, Fong L and Wang HG: Interactions among members of the Bcl-2 protein family analyzed with a yeast two-hybrid system. Proc Natl Acad Sci USA 91: 9238-9242, 1994.

12. Oltvai ZN and Korsmeyer SJ: Checkpoints of dueling dimers foil death wishes. Cell 79: 189-192, 1994.

13. Yin XM, Oltvai ZN and Korsmeyer SJ: BH1 and BH2 domains of Bcl-2 are required for inhibition of apoptosis and heterodimerization with Bax. Nature 369: 321-323, 1994.

14. Chen SJ, Wang JL, Chen JH and Huang RN: Possible involvement of glutathione and p53 in trichloroethylene- and perchloroethylene-induced lipid peroxidation and apoptosis in human lung cancer cells. Free Radic Biol Med 33: 464-472, 2002.

15. Latif N, Khan MA, Birks E, O'Farrell A, Westbrook J, Dunn MJ and Yacoub MH: Upregulation of the Bcl-2 family of proteins in end stage heart failure. J Am Coll Cardiol 35: 1769-1777, 2000.

16. Voinnet O: RNA silencing: Small RNAs as ubiquitous regulators of gene expression. Curr Opin Plant Biol 5: 444-451, 2002.

17. Hammond SM: Dicing and slicing: The core machinery of the RNA interference pathway. FEBS Lett 579: 5822-5829, 2005.

18. Ikeda S, Kong SW, Lu J, Bisping E, Zhang H, Allen PD, Golub TR, Pieske B and Pu WT: Altered microRNA expression in human heart disease. Physiol Genomics 31: 367-373, 2007. 
19. Nair N, Kumar S, Gongora E and Gupta S: Circulating miRNA as novel markers for diastolic dysfunction. Mol Cell Biochem 376: 33-40, 2013

20. Dong S, Ma W, Hao B, Hu F, Yan L, Yan X, Wang Y, Chen Z and Wang Z: microRNA-21 promotes cardiac fibrosis and development of heart failure with preserved left ventricular ejection fraction by up-regulating Bcl-2. Int J Clin Exp Pathol 7: 565-574, 2014.

21. Richardson P, McKenna W, Bristow M, Maisch B, Mautner B, O'Connell J, Olsen E, Thiene G, Goodwin J, Gyarfas I, et al: Report of the 1995 world health organization/international society and federation of cardiology task force on the definition and classification of cardiomyopathies. Circulation 93: 841-842, 1996.

22. Zhang WB, Du QJ, Li H, Sun AJ, Qiu ZH, Wu CN, Zhao G, Gong H, Hu K, Zou YZ and Ge JB: The therapeutic effect of rosuvastatin on cardiac remodelling from hypertrophy to fibrosis during the end-stage hypertension in rats. J Cell Mol Med 16 2227-2237, 2012.

23. Adams KF Jr, Dunlap SH, Sueta CA, Clarke SW, Patterson JH, Blauwet MB, Jensen LR, Tomasko L and Koch G: Relation between gender, etiology and survival in patients with symptomatic heart failure. J Am Coll Cardiol 28: 1781-1788, 1996

24. Han Y, Peters DC, Dokhan B and Manning WJ: Shorter difference between myocardium and blood optimal inversion time suggests diffuse fibrosis in dilated cardiomyopathy. J Magn Reson Imaging 30: 967-972, 2009.

25. Unverferth DV, Baker PB, Swift SE, Chaffee R, Fetters JK, Uretsky BF, Thompson ME and Leier CV: Extent of myocardial fibrosis and cellular hypertrophy in dilated cardiomyopathy. Am J Cardiol 57: 816-820, 1986.

26. Saraste A, Pulkki K, Kallajoki M, Henriksen K, Parvinen M and Voipio-Pulkki LM: Apoptosis in human acute myocardial infarction. Circulation 95: 320-323, 1997.

27. Mallat Z, Tedgui A, Fontaliran F, Frank R, Durigon M and Fontaine G: Evidence of apoptosis in arrhythmogenic right ventricular dysplasia. N Engl J Med 335: 1190-1196, 1996.
28. Boise LH, González-García M, Postema CE, Ding L, Lindsten T, Turka LA, Mao X, Nuñez G and Thompson CB: bcl-x, a bcl-2-related gene that functions as a dominant regulator of apoptotic cell death. Cell 74: 597-608, 1993.

29. Antonsson B, Conti F, Ciavatta A, Montessuit S, Lewis S, Martinou I, Bernasconi L, Bernard A, Mermod JJ, Mazzei G, et al: Inhibition of Bax channel-forming activity by Bcl-2. Science 277: 370-372, 1997 .

30. Bartel DP: MicroRNAs: Target recognition and regulatory functions. Cell 136: 215-233, 2009.

31. Thum T, Gross C, Fiedler J, Fischer T, Kissler S, Bussen M, Galuppo P, Just S, Rottbauer W, Frantz S, et al: MicroRNA-21 contributes to myocardial disease by stimulating MAP kinase signalling in fibroblasts. Nature 456: 980-984, 2008.

32. Roy S, Khanna S, Hussain SR, Biswas S, Azad A, Rink C, Gnyawali S, Shilo S, Nuovo GJ and Sen CK: MicroRNA expression in response to murine myocardial infarction: miR-21 regulates fibroblast metalloprotease-2 via phosphatase and tensin homologue. Cardiovasc Res 82: 21-29, 2009.

33. van Rooij E, Sutherland LB, Liu N, Williams AH, McAnally J, Gerard RD, Richardson JA and Olson EN: A signature pattern of stress-responsive microRNAs that can evoke cardiac hypertrophy and heart failure. Proc Natl Acad Sci USA 103: 18255-18260, 2006.

34. Shan H, Zhang Y, Lu Y, Zhang Y, Pan Z, Cai B, Wang N, Li X, Feng T, Hong Y and Yang B: Downregulation of miR-133 and miR-590 contributes to nicotine-induced atrial remodelling in canines. Cardiovasc Res 83: 465-472, 2009.

35. Engelhardt S, Hein L, Wiesmann F and Lohse MJ: Progressive hypertrophy and heart failure in beta1-adrenergic receptor transgenic mice. Proc Natl Acad Sci USA 96: 7059-7064, 1999.

36. Bisognano JD, Weinberger HD, Bohlmeyer TJ, Pende A, Raynolds MV, Sastravaha A, Roden R, Asano K, Blaxall BC, Wu SC, et al: Myocardial-directed overexpression of the human beta(1)-adrenergic receptor in transgenic mice. J Mol Cell Cardiol 32: 817-830, 2000. 